operative CA 19-9 has been used as a prognostic marker with higher levels being associated with poorer outcomes. The purpose of this study was to see if post operative Ca19-9 was an independent prognostic factor.

Methods A retrospective analysis of a prospectively collected database from January 2005 to December 2010. Inclusion criteria was a normal preoperative bilirubin and pre and postoperative Ca 19-9 measurements $(n=76)$. The primary endpoint was death or recurrence of disease. Data were also analysed for TNM staging, resection margin status and overall survival.

Results 70 patients with pancreatic ductal adenocarcinoma were in the study. An elevated post operative CA19-9 ( $n=33)$ had a significantly poor mean survival of 26.8 months compared to patients with normal levels ( $n=37$ ) who had a mean survival of 45.5 months $(p=0.004)$. For patients with a postoperative value $\geq 200 \mathrm{U} / \mathrm{ml}$ $(n=13)$ mean survival was 19.8 months compared with levels $<200$ $\mathrm{U} / \mathrm{ml}(\mathrm{n}=57)$ being 43.9 months $(\mathrm{p}=0.001)$. A $<75 \%$ fall in post operative ca19-9 levels in comparison to preoperative levels (45 vs 25 patients) resulted in poor mean survival of 34.9 vs 45.9 months but did not reach statistical significance $(\mathrm{p}=0.218)$.

Conclusion In patients who have undergone pancreaticoduodenectomy for ductal adenocarcinoma having a normal postoperative $\mathrm{Ca}$ 19-9 is a marker for improved outcome where as a level in excess of $200 \mathrm{U} / \mathrm{ml}$ is a negative predictive factor. $\mathrm{A}<75 \%$ fall in post operative readings of CA19-9 results in poor survival (11 months) but was not statistically significant.

Competing interests None declared

\section{PM0-104 THE USE OF FAECAL ELASTASE IN A DISTRICT GENERAL HOSPITAL}

doi:10.1136/gutjnl-2012-302514b.104

${ }^{1} \mathrm{E}$ Tash, ${ }^{*}{ }^{1} \mathrm{~J}$ Subhani, ${ }^{1} \mathrm{D}$ Lindo, ${ }^{1} \mathrm{D}$ Gertner, ${ }^{1} \mathrm{M}$ Joy, ${ }^{1} \mathrm{R}$ Carrigan, ${ }^{2} \mathrm{~T}$ Everitt, ${ }^{1} \mathrm{P}$ Munuswamy. 'Department of Gastroenterology, Basildon and Thurrock University Hospitals NHS Foundation Trust, London, UK; ${ }^{2}$ Department of Clinical Biochemistry, Basildon and Thurrock University Hospitals NHS Foundation Trust, London, UK

Introduction Faecal elastase $1(\mathrm{FE})$ is a proteolytic enzyme secreted by the acinar cells of the pancreas. Its determination is a highly sensitive and specific tubeless pancreatic function test. The 2003 BSG guidelines were compiled to minimise investigations and maximise positive diagnoses for patients with diarrhoea lasting more than 4 weeks. ${ }^{1}$ We analysed the appropriate use of the faecal elastase test and its correlation with symptoms in a large district general hospital.

Methods This retrospective study included all patients who had a FE requested from April 2009 to March 2010. Data were analysed for indication, symptoms, outcome of the test, follow-up, other investigations and the use of creon.

Results Over a period of 1 year, 121 patients had a FE requested. Patient notes and laboratory information was only available for a total of 101 patients. Data were collected from these notes for further analysis. 19 patients (19\%) had pancreatic insufficiency with low FE levels (ranging $<15-144$ ). 82 patients had normal FE levels $(>500)$. In patients with a low FE levels, 12 patients had diarrhoea $(63 \%)$, steatorrhoea $(21 \%)$, abdominal pain $(26 \%)$, weight loss $(47 \%)$, alcohol history $(15 \%)$, history of pancreatitis in $(53 \%)$ and none of these patients had abdominal distention, flatulence or offensive stools. $58 \%$ in the low FE group had treatment with creon. A logistic regression analysis was performed on three symptoms; diarrhoea, steatorrhoea and previous history of pancreatitis. History of pancreatitis was statistically significant with an OR of 10.21 , for faecal elastase insufficiency.

Conclusion In our study group we found that a previous history of pancreatitis was a strong predictor of a low faecal elastase. Though statistically not significant, patients with diarrhoea, steatorrhoea and weight loss, do benefit from FE testing.

Competing interests None declared.

\section{REFERENCE}

1. Thomas PD, et al. Guidelines for the investigation of chronic diarrhoea. 2nd edn. Gut 2003;52(Suppl V):v1-5.

\section{PM0-105 PANCREATIC PSEUDOCYST MANAGEMENT: EXPERIENCE FROM A DGH CENTRE}

doi:10.1136/gutjnl-2012-302514b.105

J Kavanagh, ${ }^{*}$ R Sringeri, S Shetty. Department of Gastroenterology, The Dudley Group NHS Foundation Trust, Birmingham, UK

Introduction Pancreatic pseudocysts are complications of acute or chronic pancreatitis. Diagnosis is usually accomplished by cross sectional imaging and cyst fluid analysis will help in differentiating pseudocyst from other cystic lesions of pancreas. Most of them resolve with supportive care but intervention is required if there are persistent symptoms or develop any complications. ${ }^{1}$ Our aim was to review the practice of pancreatic pseudocyst management in a DGH set up and to formulate a care pathway or protocol supported by good evidence base.

Methods Retrospective case notes review of patients diagnosed with pancreatic pseudocyst. Patients were identified by ICD classification based clinical coding database. Data collected includes patient demographics, aetiology of pancreatitis, size and number of cysts, diagnostic modalities used, associated symptoms and complications, indication for intervention and type of intervention used and follow-up strategy

Results 62 patients were diagnosed to have pancreatic pseudocyst in last 7 years. Median age 60 yrs (range 24-91 yrs), male to female ratio was $1.4: 1$. Total of $81 \%(n=50)$ had history of acute pancreatitis. Alcohol $(n=32,50 \%)$ was the commonest underlying aetiology. Abdominal pain was the commonest symptom ( $\mathrm{n}=55,89 \%)$ and $10 \%(n=6)$ were asymptomatic. CT scan was used as diagnostic modality in $90 \%(n=56)$ and USS in $74 \%(n=46)$. Total of $82 \%$ $(n=51)$ had a single cyst where as $18 \%(n=11)$ had multiple. Average cyst size was $70 \mathrm{~mm}$ (range $10-270 \mathrm{~mm}$ ). About 76\% (45/59, three died) had follow-up scan and average duration of follow-up was 13 months. Of them, cyst got increased in size in $32 \%(n=15)$, decreased in size in $23 \%(n=11)$, no change in $28 \%(n=11)$ and got resolved in $17 \%(n=8)$. Total of $43 \%(n=23)$ had their symptoms resolved spontaneously without requiring any intervention. Complications were noted in $28 \%$ ( $n=17$, CBD obstruction-7, cyst infection-6, gastric/duodenal obstruction-3 and cyst rupture in 1). About $39 \%(n=24)$ required intervention because of persistent symptoms or due to complications. Persisting pain $(n=18,29 \%)$ and increase in cyst size $(\mathrm{n}=7,11 \%)$ were the commonest indications. In $58 \%(n=14)$ endoscopic, 33\% $(n=8)$ percutaneous and $8 \%(2)$ required surgical drainage. Of those who had intervention, $79 \%(n=19)$ required once, $17 \%(n=4)$ twice and $4 \%(n=1)$ thrice. Overall $79 \%$ $(19 / 24)$ had symptoms resolved post intervention.

Conclusion Pancreatic pseudocysts were managed conservatively in more than half of the patients group. In nearly one fifth of patients spontaneous resolution of cysts were noted in follow-up scans. Those who required intervention, endoscopic method was the most common method used and many patients achieved good resolution of their symptoms.

Competing interests None declared.

\section{REFERENCES}

1. Andren Sandenberg A, et al. JOP 2004;5:8-24. 